\title{
Maintaining the Genome
}

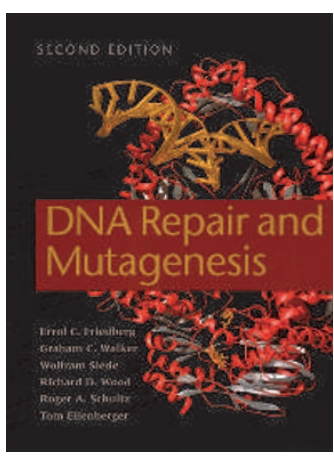

\section{DNA Repair and Mutagenesis (Second Edition)}

\begin{abstract}
By Errol Friedberg, Graham Walker, Wolfram Siede, Richard Wood, Roger Schultz and Tom Ellenberger
\end{abstract}

ASM Press $\bullet 2006 \cdot \$ 175.00$

\section{Philip C. Hanawalt}

The remarkable genetic continuity from generation to generation was once thought to be solely the consequence of a unique stability of the chromosomal substance, as Erwin Schrödinger declared in 1944 in his classic monograph What is life? Ten years later Watson and Crick proposed the double-helical structure for DNA and postulated a mechanism for its replication. Within another decade it was revealed that the complementary base pairing and inherent redundancy of information in duplex DNA could also be employed for correcting errors or damage affecting either strand. We now appreciate that the genome is quite pliable stuff and that DNA is not an unusually stable chemical entity. DNA suffers a continuous barrage of disrupting events from environmental radiations or genotoxic chemicals, endogenous reactive oxygen species, and chemical decomposition. Life would be impossible were it not for a remarkable variety of sensors and well-coordinated DNA repair pathways that continually monitor the genome for irregularities and process them in a manner appropriate to essential cellular function.

It is telling that the heaviest book (both in weight and in content!) on my desk is the comprehensive treatise on DNA repair that Errol Friedberg and his five industrious coauthors have recently brought to fruition. This volume is the much-expanded and largely rewritten second edition of a text that three of these authors published ten years ago. The completion of a text in this field presented a rapidly-moving target for the authors as the continual flood of new information threatened the project with obsolescence by the time proofs could be corrected. They have dealt effectively with this problem by taking a balanced historical approach that includes input from different laboratories and relies on DNA-repair models that are well-substantiated, together with many that are still preliminary. The flow of investigative research is documented through an amazingly complete list of over 10,000 references. This, at first, gives the impression that the intent was to cite every published paper in the field, but the authors have, in fact, been quite selective in their choice of references. The book is a highly informative resource for

Philip C. Hanawalt is in the Department of Biological Sciences, Stanford University, Stanford, CA 94305, USA. e-mail: hanawalt@stanford.edu every known DNA-repair system, including several discovered within the past few years. It also gives the impression of a vibrant field with many remaining problems to be solved.

The 30 chapters have been incorporated into five logical sections and the 700+ figures include remarkably informative crystal structures and molecular models - especially considering that only two colours were used. A CD is available with the figures and tables and is a useful aide for instructors.

Because of the interrelatedness and overlap of multiple pathways for processing damaged DNA, it is a challenge to present the topics in a logical order. Extensive crossreferencing in the introductory sections of most chapters helps to solve this problem. The predominant emphasis throughout the book is on the experimental basis for understanding mechanisms of mutagenesis and DNA repair. The level of detail is informative for students who wish to learn the appropriate research methodologies as they enter the field. A very useful table in the appendix clarifies DNA-repair gene nomenclature for similar enzymes from the principal organisms studied.

The book is a highly informative resource for every known DNA-repair system, including several discovered within the past few years...

There is some lack of uniformity in the text through the successive chapters, largely due to the stylistic preferences of the six authors. Thus, the text is not as "seamlessly integrated" as was claimed in the preface as a goal. Only half of the chapters end with a summary and this is used in quite different ways - in some cases to provide a synopsis, but in others to indicate unsolved problems and where the respective subfields are headed.

I subjected the freshly minted text to the acid-test of student critiques during my graduate course on "DNA repair and genomic stability". Each student was assigned a chapter to review, including comment on its pedagogical value. Responses were generally positive and most students appreciated the emphasis on experimental approaches. Some felt that their chapter was more like a mini-review than a textbook chapter. One commented that it reads more like an encyclopaedia or reference work than a textbook. Several complained that important and less-important facts were not clearly delineated and that general principles were sometimes obscured by too much detail. The students, of course, took delight in finding minor errors in the text, such as incorrect reference citations or typographical errors. I found remarkably few mistakes during my own readings, although there were points where my interpretations were at variance with those of the authors. Many students commented that the book was too lengthy to serve as a text for a one-quarter course, but that it was extremely useful as a reference for preparing their term papers on a detailed topic in the field of DNA repair and mutagenesis. That was also my own conclusion by the end of the course.

The authors have performed a remarkable service for the DNA-repair community and for those in many other areas who wish to learn why we find this field so exciting and relevant. The volume will stand as the classic reference on DNA repair and mutagenesis for a long time to come. 